3 Research Square

\title{
Impact of sex differences on lifestyle and glycemic control in Japanese patients with diabetes during COVID-19 lockdowns
}

Junpei Shikuma ( $\nabla$ shikuma@tokyo-med.ac.jp)

Tokyo Medical University https://orcid.org/0000-0001-6531-2388

\section{Yoshiyuki Nagai}

Tokyo Medical University: Tokyo Ika Daigaku

Mamoru Sakurai

Tokyo Medical University: Tokyo Ika Daigaku

Kana Udagawa

Tokyo Medical University: Tokyo Ika Daigaku

Rokuro Ito

Tokyo Medical University: Tokyo Ika Daigaku

\section{Takashi Miwa}

Tokyo Medical University

\section{Ryo Suzuki}

Tokyo Medical University

\section{Research Article}

Keywords: COVID-19, Diabetes, Lifestyle, Sex differences

Posted Date: October 18th, 2021

DOl: https://doi.org/10.21203/rs.3.rs-965006/v1

License: (9) This work is licensed under a Creative Commons Attribution 4.0 International License.

Read Full License 


\section{Abstract \\ Background}

Pneumonia of unknown pathogenesis detected in Wuhan, Hubei Province, China, in December 2019 was later found to be a new coronavirus infection that causes the now-named coronavirus disease 2019 (COVID-19). The onset of the pandemic has drastically changed global lifestyles. Some reports about lifestyle changes in patients with diabetes during COVID-19 pandemic have been published. However, these studies have not assessed sex differences. Thus, we analyzed three lifestyle changes during the COVID-19 pandemic to determine any sex differences.

\section{Methods}

We analyzed physical activity, snacking habits, and drinking habits in 323 patients with diabetes. Sex differences in the changes in these three lifestyle habits were analyzed using the $\chi^{2}$ test, and the factors that influenced the deterioration of $\mathrm{HbA} 1 \mathrm{c}$ were analyzed by paired t-test. The factors that influenced the deterioration of $\mathrm{HbA1c}$ were determined by univariate and multivariate logistic regression analyses.

\section{Results}

Of the 323 diabetic, 212 were male (65.6\%) and 111 were female (34.4\%). HbA1c values were higher in 2020 compared with those in 2019. Increased physical activity was higher in men, whereas decreased physical activity was higher in women. The factors that affected the deterioration in $\mathrm{HbA} 1 \mathrm{c}$ were snacking habits for the overall population and in the male sex. No factors significantly affected HbA1c in the female population.

\section{Conclusions}

Our study confirmed that lifestyle changes differed between the sexs during the COVID-19 pandemic. Snacks influenced the deterioration of $\mathrm{HbA} 1 \mathrm{c}$ in the overall study population and in the male population. A balanced diet and regular exercise are important for all patients with diabetes during this pandemic. This study demonstrates that more attention should be given on men's diet, whereas physical inactivity should be emphasized for women.

\section{Background}

Pneumonia of unknown pathogenesis was documented in Wuhan, Hubei Province, China, in December 2019 and was later identified as a new coronavirus infectious disease now-named coronavirus disease 2019 (COVID-19) [1]. The disease has since spread rapidly worldwide. On January 14, 2020, a patient with pneumonia who had stayed in Wuhan City was the first case reported in Japan [2]. In China and some 
Western countries, where the number of infected people had first spread rapidly, strong measures, such as city lockdowns, were imposed. In Japan, schools were temporarily closed from February 28, 2020, and teleworking was recommended for companies. Based on the Act on Special Measures Low for COVID-19, which was enacted on March 13, 2020, a state of emergency was issued in Japan on April 7. The Japanese people were requested to refrain from going out unnecessarily, and the declaration lasted until May 25. Such declarations were issued repeatedly thereafter, and this has changed the people's lifestyles drastically.

Diabetes is a typical lifestyle-related disease, and lifestyle habits greatly influence the management of such diseases. Changes in the lifestyles of patients with diabetes have due to the COVID-19 pandemic have been reported [3-7]. Some studies found deterioration in lifestyle-related habits such as dieting, snacking habits, and exercise; increased stress; and an association between glycemic control and weight gain. Other studies reported no changes in body weight or $\mathrm{HbA1c}$, but found an association between mental stress and lack of sleep with unhealthy eating habits, resulting in poor glycemic control in the study populations. However, these reports have not evaluated sex differences in lifestyle changes due to the COVID-19 pandemic. As an example of sex differences in living with diabetes, women may neglect their own health by prioritizing their household chores and family [8]. We therefore sought to bridge the gap and retrospectively analyzed three basic lifestyle-related changes during the COVID-19 pandemic to determine whether any sex differences exist and whether they are related to glycemic control.

\section{Methods}

We enrolled 323 patients with diabetes who were under regular follow-up and collected data by direct interview on changes in three subjective lifestyle habits (physical activity, snacking habits, and drinking habits) due to the COVID-19 pandemic during their visit to our hospital. Inclusion criteria were patients with diabetes who were visiting our hospital aged 20 years old and above. Any type of diabetes was included. Exclusion criteria were patients who could not answer our interview independently. The three lifestyle habits (physical activity, snacks, and drinking) were analyzed according to three stages, namely, decrease, unchanged, and increase. The categorization was based on the patient's subjective evaluation. In addition to changes in the three lifestyle habits, data on sex, age, employment status (telework status), and $\mathrm{HbA} 1 \mathrm{c}$ were extracted from the medical records. The $\mathrm{HbA} 1 \mathrm{c}$ values obtained were the quarterly values for January-March, April-June, July-September, and October-December and the annual average values for 2019 and 2020. In calculating the rate of change in the annual average $\mathrm{HbA1c}$ (2020 average $\mathrm{HbA} 1 \mathrm{c}$ to 2019 average $\mathrm{HbA} 1 \mathrm{c}$ ), $0 \%$ or less was defined as the improvement group, and $0.1 \%$ or more was defined as the deterioration group. As this study was a retrospective study, we did not need to obtain informed consent. Instead, we presented an opt-out on our website that explained the following: $\mathbb{V}$ purpose of use and method of use of information, $\nabla$ items of information to be used, $\nabla$ scope of users, $\nabla$ name of the person responsible for managing information $\nabla$ exclusion of information that identifies the research subject at the request of the research subject or his/her agent, and $\nabla$ process of accepting the request of the research subject or his/her agent. This study was conducted with the approval of the Medical Ethics Review Board of Tokyo Medical University (Approval Code T2020-0381). 


\section{Analysis 1}

We assessed for sex differences the changes in three lifestyle habits caused by the COVID-19 pandemic.

\section{Analysis 2}

The factors that affected the deterioration of the mean $\mathrm{HbA} 1 \mathrm{c}$ value were analyzed by sex by univariate and multivariate analyses.

\section{Statistical analysis}

The comparison of HbA1c values in 2019 and 2020 was analyzed by paired t-test. Sex differences in the changes in the three lifestyle habits were analyzed using the $\chi^{2}$-square test. Univariate and multivariate logistic regression analyses were used to analyze the data of the $\mathrm{HbA} 1 \mathrm{c}$ improvement and deterioration groups. The outcome was the improvement/deterioration of the mean $\mathrm{HbA} 1 \mathrm{c}$ value, and the explanatory variables were age, employment status, and changes in the three lifestyle habits.

\section{Results}

Of the 323 patients with diabetes, 212 were male (65.6\%) and 111 were female (34.4\%). Twenty-four patients had type 1 diabetes (7.4\%), 291 patients had type 2 diabetes (90.1\%), and 8 patients had another type of diabetes (2.5\%). The mean age was $63.1 \pm 13.1$ years (male, $62.7 \pm 11.8$ years; female, $63.8 \pm$ 15.3 years) (Table 1). The annual mean $\mathrm{HbA} 1 \mathrm{c}$ value in 2019 was $7.6 \pm 1.1 \%$ (male, $7.6 \% \pm 1.1 \%$; female, $7.5 \% \pm 1.6 \%$ ), whereas the annual mean $\mathrm{HbA} 1 \mathrm{c}$ value in 2020 was $7.6 \pm 1.0 \%$ (male, $7.6 \% \pm 1.0 \%$; female $7.6 \% \pm 1.0 \%$ ). No significant differences in the variables were found between the two years. When examined by quarter, the $\mathrm{HbA1c}$ levels increased significantly in 2020 compared with 2019 in the JulySeptember period. (July-September HbA1c 2019 vs. 2020: 7.4\% $\pm 1.0 \%$ vs. $7.6 \% \pm 1.1 \% ; p=0.000058$ ) This tendency was observed in both sexes. (Male: $7.4 \pm 1.0$ vs. $7.6 \pm 1.1, p=0.001$; Female: $7.4 \pm 1.1$ vs. $7.7 \pm 1.1, p=0.006)$ (Fig. 1). 
Table 1

Patients' characteristics

\begin{tabular}{|lll|}
\hline Subjects & \multicolumn{2}{l|}{323} \\
\hline Mean age & $63.1 \pm 13.1$ \\
\hline & Number & $\%$ \\
\hline Male & 212 & 65.6 \\
\hline Female & 111 & 34.4 \\
\hline Type 1 diabetes & 24 & 7.4 \\
\hline Type 2 diabetes & 291 & 90.1 \\
\hline Other type of diabetes & 8 & 2.5 \\
\hline Diabetes medication & & \\
\hline Biguanide & 192 & 59.4 \\
\hline Thiazolidine & 2 & 0.6 \\
\hline aGlucosidase inhibitor & 20 & 6.2 \\
\hline SGLT2 inhibitor & 111 & 34.4 \\
\hline DPP4 inhibitor & 180 & 55.7 \\
\hline GLP-1 receptor agonist & 34 & 10.5 \\
\hline Sulfonylurea & 47 & 14.6 \\
\hline Glinide & 22 & 6.8 \\
\hline Insulin & 93 & 28.8 \\
\hline
\end{tabular}

\section{Analysis 1: Sex differences in changes in three lifestyle habits}

Overall, for physical activity, the frequency of decrease was $47 \%$, unchanged was $41 \%$, and increase was $12 \%$. Sex differences were observed in the frequency of changes in physical activity $\left(\chi^{2}(2)=13.6 ; p=\right.$ 0.001 (Male vs. Female, Decreased/Unchanged/Increased: 40\% /44\%/16\% vs. 60\%/35\%/5\%; $p=0.001$ ). In the residual analysis, the frequency of increased physical activity was significantly higher in men, and the frequency of decreased physical activity was significantly higher in women. Overall, for snacking habits, frequency of decreased was $6 \%$, unchanged was $62 \%$, and increase was $32 \%$. Sex differences were observed in the frequency of changes in snacking habits $\left(\chi^{2}(2)=7.67 ; p=0.022\right)$ (Male vs. Female, Decreased/Unchanged/Increased: $5 \% / 67 \% / 28 \%$ vs. $10 \% / 52 \% / 38 \% ; p=0.001)$. In the residual analysis, only the frequency of unchanged snacking habits was significantly higher in men. Overall, for drinking habits, the frequency of decrease was $14 \%$, unchanged was $80 \%$, and increase was $6 \%$. Sex differences 
were observed in the frequency of changes in drinking habits $\left(\chi^{2}(2)=14.8 ; p=0.001\right.$ ) (Male vs. Female, Decreased/Unchanged/Increased: $17 \% / 74 \% / 9 \%$ vs. $6 \% / 92 \% / 2 \% ; p=0.001)$. In the residual analysis, the frequency of increase and decrease in drinking habits were significantly higher in men. The frequency of unchanged was significantly higher in women (Fig. 2).

\section{Analysis 2: Factors affecting the deterioration of the mean $\mathrm{HbA1c}$ value}

No significant associations were observed in age, telework, drinking habits, physical activity, and the deterioration of the mean $\mathrm{HbA1c}$ value. Snacking habits were significantly associated with the deterioration of the mean $\mathrm{HbA} 1 \mathrm{c}$ value in the overall analysis (odds ratio [OR] 1.704, 95\% confidence interval [Cl]: 1.126-2.579) and with the male sex (OR 1.879, 95\% Cl: 1.063-3.320) in multivariate analysis. Employment status was also significantly associated with the male sex (OR $3.216,95 \% \mathrm{Cl}$ : 1.605-6.443) analysis. On the other hand, in the female population, although no factors were significantly associated with changes in the $\mathrm{HbA} 1 \mathrm{c}$ values, univariate analysis showed that unemployed women tended to exhibit a deterioration in $\mathrm{HbA1c}$ values (OR $0.492,95 \% \mathrm{Cl}: 0.228-1.06 ; \mathrm{p}=0.07$ ) Interestingly, the opposite trend was observed in the male population. Among the employed people, no significant association was observed between telework and the deterioration of mean $\mathrm{HbA} 1 \mathrm{c}$ in the overall population and in both the male and female populations (Table 2).

Table 2

Univariate and multivariate logistic regression analysis of deterioration of mean $\mathrm{HbA} 1 \mathrm{c}$

\section{Deterioration of mean $\mathrm{HbA1c}$}

Univariate significant variables $(\mathrm{p}<$ 0.05)

Multivariate significant variables $(p<0.05)$

$\begin{array}{lllll}\text { Overall } & \text { OR }(95 \% \mathrm{Cl}) & \mathrm{p} \text { value } & \mathrm{OR}(95 \% \mathrm{Cl}) & \mathrm{p} \text { value } \\ \text { work } & 1.431(0.915-2.236) & 0.116 & 1.540(0.930-2.551) & 0.093 \\ \text { snack } & 1.762(1.175-2.642) & 0.006 & 1.704(1.126-2.579) & 0.012\end{array}$

Male

$\begin{array}{lllll}\text { work } & 2.667(1.499-4.744) & 0.001 & 3.216(1.605-6.443) & 0.001\end{array}$

$\begin{array}{lllll}\text { snack } & 1.925(1.116-3.321) & 0.019 & 1.879(1.063-3.320) & 0.03\end{array}$

Female

$\begin{array}{lllll}\text { work } & 0.492(0.228-1.060) & 0.07 & 0.483(0.210-1.108) & 0.86\end{array}$

$\begin{array}{lllll}\text { snack } & 1.575(0.858-2.891) & 0.143 & 1.674(0.870-3.220) & 0.123\end{array}$

\section{Discussion}


Due to the COVID-19 pandemic, measures such as city lockdowns were imposed across the world. In Japan, people were mandated to refrain from going out unnecessarily and to work from home when possible, schools were closed. This has given people more time to spend at home and has associated physical, mental, and social stress. Many reports have been published on how these lockdowns affect lifestyle habits. An online survey of 1,033 Chinese people showed that $70 \%$ of participants increased their screen time, whereas $30 \%$ of participants increased in the frequency of their vegetable and fruit intake [9]. An analysis of 995 Indian people revealed that physical activity decreased, whereas sitting time and screen time increased. Improvement in dietary habits was seen in younger people. Body weights increased in one-third of participants. Quarantine-induced stress and anxiety increased in nearly onefourth of the participants [10]. A study on young Saudi women (aged 19-30) showed that approximately half of the participants did not report a weight change, $30 \%$ reported weight loss, and $18 \%$ reported weight gain. The variables associated with increased weight gain were self-quarantine since COVID-19 started, age, and stress at baseline and during lockdown. The variables associated with reduced risk of weight gain were physical activity during lockdown, coffee consumption, and total sleep time [11]. According to a survey conducted at 35 research institutes in West Asia, North Africa, Europe, and the United States, which used the Internet to evaluate the psychological and lifestyle-related effects of home confinement due to COVID-19, the period of home confinement had a negative effect on mental health and emotions, and these were associated with unhealthy lifestyle (lack of physical activity $+15.2 \%$; decreased social activity $+71.2 \%$; deterioration of sleep quality $+12.8 \%$; unhealthy eating habits $+10 \%$ compared with the period before confinement) [12]. In the general population, physical inactivity has been identified in many reports, although dietary habits varied. Many reports showed that increased stress leads to unhealthy eating habits. In a study on patients with diabetes in India, about $80 \%$ of people reported that their diet and physical activity remained unchanged. Body weight and $\mathrm{HbA} 1 \mathrm{c}$ were unchanged, but mental stress and lack of sleep were associated with unhealthy eating habits, resulting in poor glycemic control in these populations. The importance of a mental stress approach was emphasized [7].

Four reports in Japan observed a relationship between lifestyle changes and glycemic control due to the COVID-19 pandemic. Munekawa et al. [3] investigated stress levels and lifestyle-related changes in 203 patients with type 2 diabetes using a questionnaire and found that $40 \%$ of the study population felt increased stress, and $50 \%$ had decreased physical activity. Increased stress was associated with decreased physical activity and increased dietary intake. Tanaka et al. [4] conducted a self-administered questionnaire survey of 463 patients with diabetes. They found an increase in snacking habits in $21 \%$, a decrease in physical activity in $51.2 \%$, and an increase in alcohol intake in $8.7 \%$. People under the age of 65 years with increased snacking exhibited worsening $\mathrm{HbA} 1 \mathrm{c}$ and weight gain, whereas in those over the age of 65 years, physical inactivity was a factor of weight gain. A difference depending on the age has been reported. Takahara et al. [5] observed the relationships among lifestyle-related changes, HbA1c, and body weight in 1402 patients with diabetes using an interview questionnaire. A decrease in leisure time exercise as associated with worsening $\mathrm{HbA} 1 \mathrm{c}$ and weight gain, whereas an increase in dietary intake and snacking were each associated with weight gain. Differences in these relationships were found to vary 
with age and with exercise habits. Kishimoto et al. [6] surveyed 168 diabetic patients. Patients with reduced exercise due to teleworking or gym closure had worse glycemic control, but patients with improved diet, regardless of exercise, reported improved glycemic control. In this study, $32 \%$ showed increased snacking, whereas $47 \%$ showed decreased physical activity. In the overall analysis, the factor statistically associated with worsening glycemic control was increased snacking, indicating the importance of diet management during the COVID-19 pandemic, as demonstrated by Kishimoto et al.

In this study, we examined the factors that influence the deterioration of HbA1c during the COVID-19 pandemic according to sex to determine whether lifestyle changes differed according to sex. In the male population, many of the employed had worsened $\mathrm{HbA} 1 \mathrm{c}$, whereas no association between employment status and worsened $\mathrm{HbA1c}$ was observed. However, the unemployed subjects exhibited worsened $\mathrm{HbA1c}$, and a sex difference was observed.

Sex differences were observed in the changes in the three lifestyle habits (physical activity, snacking habits, and drinking habits). Comparing the physical activity between the sexes especially indicated that it decreased among the women answered but increased among the men. Thus, women may be more likely to be less physically active than men during the pandemic.

Much research has been done on sex differences in normal conditions, but not during the COVID-19 pandemic. According to demographic and behavioral data from a cohort study in 20 countries, the "PURESaudi" study, women were more likely to experience some form of stress [13]. In real life, women may neglect their own health by prioritizing their household chores and family [8]. Since ancient times, men have been hunting, and women have been collecting to secure food. Men and women in that period were said to vary in their dietary behaviors. The Handza tribe of Tanzania still maintain these traditional, sexbased hunter-gatherer roles. Reports on the differences in dietary behaviors between the men and women of the Handza stated that males consumed a higher proportion of meat in total calories, whereas females consumed a higher proportion of tubers (plant roots). Women were also reported to eat more often than men [14].

In addition, while women are highly interested in healthy eating and form their own weight and eating habits, men tend to eat fatty foods because they prioritize the joy of eating [15]. These sex differences in eating behavior are influenced by sex hormones. Estrogen, a female hormone, is said to have anti-obesity and anti-feeding properties. Chronic increased stress is known to cause a bias toward a high-energy diet (rich in sugars and lipids), which is said to be more pronounced in men [16]. The cortisol-secreting response to stress may increase food intake and sweets [17]. Unproductive time or spells of boredom also affects eating behavior. Boredom is known to increase the desire for snacking, which is considered an avoidance behavior from self-loathing caused by boredom [18]. Chronic psychological stress and boredom as a result of lockdowns during the COVID-19 pandemic, therefore, increase the tendency for a high-energy dietary intake and snacking, and the effects can be significant, especially in men. The present study also indicates that snacking contributes to the deterioration of HbA1c in men. The effects of lifestyle-related changes on $\mathrm{HbA} 1 \mathrm{c}$ were associated with an increase in snacking in the overall and male 
population analyses. Patients with diabetes need to maintain balanced diet and regular exercise during the COVID-19 pandemic period. Furthermore, this study emphasized the need for glycemic control in men with diabetes, whereas the need to engage in physical activity was identified in women.

The data obtained on the changes in lifestyle habits were not quantitative evaluations but were subjective responses of the subjects. No dietary intake or nutritional survey other than snacking was investigated. Patient information such as complications and duration of diabetes were not sufficiently investigated. In addition, because this study is a retrospective medical record survey, the medications were administered at the discretion of the attending physician and thus varied among the patients. These limitations must be thoroughly addressed in future studies to obtain more conclusive results.

\section{Conclusion}

This study found sex differences in lifestyle changes in patients with diabetes during the COVID-19 pandemic. The findings suggest that treatment for diabetes should be tailored in consideration of sex differences.

\section{Abbreviations}

COVID-19, coronavirus disease 2019

\section{Declarations}

\section{Ethics approval and consent to participate}

This retrospective study was approved by the Medical Ethics Review Board of Tokyo Medical University (Approval Code: T2020-0381).

\section{Consent for publication}

Not applicable.

\section{Availability of data and material}

The datasets analyzed during this study are available from the corresponding author on reasonable request.

\section{Competing interests}

The authors have no conflicts of interest to declare.

\section{Funding}

Authors did not receive any funding for this work. 


\section{Authors' contributions}

Conception or design: JS and YN. Acquisition, analysis, or interpretation of data: JS, YN, MS, KU, and RI. Discussion and writing this manuscript: JS and RI. Supervision of entire this research: TM and RS.

\section{Acknowledgements}

We thank Dr. Ayaka Oguchi, Dr. Maya Takahashi, and Dr. Aya Kondo for collecting data from medical records.

\section{References}

1. Wang C, Horby PW, Hayden FG, Gao GF. A novel coronavirus outbreak of global health concern. Lancet. 2020;395:470-3.

2. Kato T, Yanagawa M. Pneumonia associated with covid-19 (1). Ministry of Health, Labour and Welfare newsletter, Japan. 2020. https://www.mhlw.go.jp/stf/newpage_08906.html. Accessed 1 Aug 2021.

3. Munekawa C, Hosomi Y, Hashimoto Y, Okamura T, Takahashi F, Kawano R, et al. Effect of coronavirus disease 2019 pandemic on the lifestyle and glycemic control in patients with type 2 diabetes: a cross-section and retrospective cohort study. Endocrine J. 2021;68:201-10.

4. Tanaka N, Hamamoto Y, Kurotobi Y, Yamasaki Y, Nakatani S, Matsubara M, et al. Lifestyle changes as a result of COVID-19 containment measures: bodyweight and glycemic control in patients with diabetes in the Japanese declaration of a state of emergency. J Diabetes Investig. 2021;12:1718-22.

5. Takahara M, Watanabe H, Shiraiwa T, Maeno Y, Yamamoto K, Shiraiwa Y, et al. Lifestyle changes and their impact on glycemic control and weight control in patients with diabetes during the coronavirus disease 2019 pandemic in Japan. J Diabetes Investig. 2021. doi:10.1111/jdi.13555.

6. Kishimoto M, Ishikawa T, Odawara M. Behavioral change in patients with diabetes during the COVID19 pandemic. Diabetol Int. 2021;12:241-5.

7. Sankar P, Ahmed WN, Koshy VM, Jacob R, Sasidharan S. Effects of COVID-19 lockdown on type 2 diabetes, lifestyle and psychosocial health: a hospital-based cross-sectional survey from South India. Diabetes Metab Syndr Clin Res Rev. 2020;14:1815-9.

8. Siddiqui MA, Khan MF, Carline TE. Sex differences in living with diabetes mellitus. Mat Soc Med. 2013;25:140-2.

9. Hu Z, Lin X, Kaminga AC, Xu H. Impact of the COVID-19 epidemic on lifestyle behaviors and their association with subjective well-being among the general population in mainland China: crosssectional study. J Med Internet Res. 2020;22:e21176.

10. Chopra S, Ranjan P, Singh V, Kumar S, Arora M, Hasan MS, et al. Impact of COVID-19 on lifestylerelated behaviours- a cross-sectional audit of responses from nine hundred and ninety-five participants from India. Diabetes Metab Syndr Clin Res Rev. 2020;14:2021-30. 
11. Al-Musharaf S, Aljuraiban G, Bogis R, Alnafisah R, Aldhwayan M, Tahrani A. Lifestyle changes associated with COVID-19 quarantine among young Saudi women: A prospective study. PloS One. 2021;16:e0250625.

12. Ammar A, Trabelsi K, Brach M, Chtourou H, Boukhris O, Masmoudi L, Bouaziz B, Bentlage E, How D, Ahmed M, Mueller P. Effects of home confinement on mental health and lifestyle behaviours during the COVID-19 outbreak: insight from the ECLB-COVID19 multicenter study. Biol Sport. 2021;38:9-21.

13. Alhabib KF, Batais MA, Almigbal TH, Alshamiri MQ, Altaradi H, Rangarajan S, et al. Demographic, behavioral, and cardiovascular disease risk factors in the Saudi population: results from the Prospective Urban Rural Epidemiology study (PURE-Saudi). BMC Public Health. 2020;20:1213.

14. Berbesque JC, Marlowe FW, Crittenden AN. Crittenden. Sex differences in Hadza eating frequency by food type. Am J Hum Biol. 2011;23:339-45.

15. Grzymisławska M, Puch EA, Zawada A, Grzymisławski M. Do nutritional behaviors depend on biological sex and cultural sex? Adv Clin Exp Med. 2020;29:165-72.

16. Torres SJ, Nowson CA. Relationship between stress, eating behavior, and obesity. Nutrition. 2007;23:887-94.

17. Epel E, Lapidus R, McEwen B, Brownell K. Stress may add bite to appetite in women: a laboratory study of stress-induced cortisol and eating behavior. Psychoneuroendocrinology. 2001;26:37-49.

18. Moynihan AB, van Tilburg WAP, Igou ER, Wisman A, Donnelly AE, Mulcaire JB. Eaten up by boredom: consuming food to escape awareness of the bored self. Front Psychol. 2015. doi:10.3389/fpsyg.2015.00369.

\section{Figures}




\section{Total}

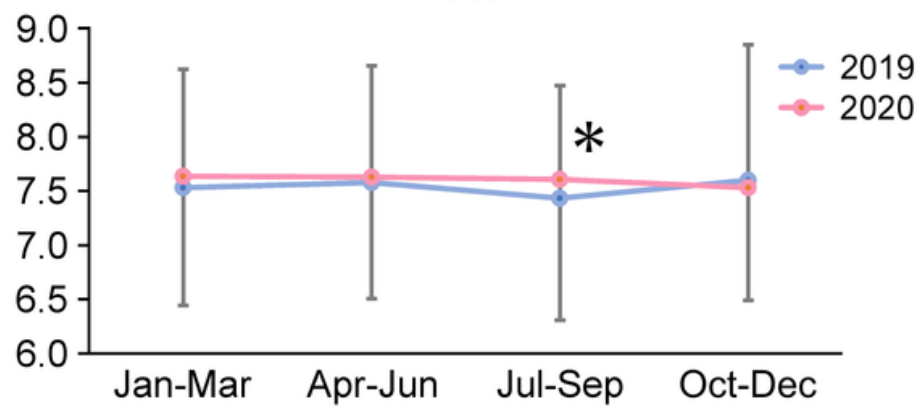

Male

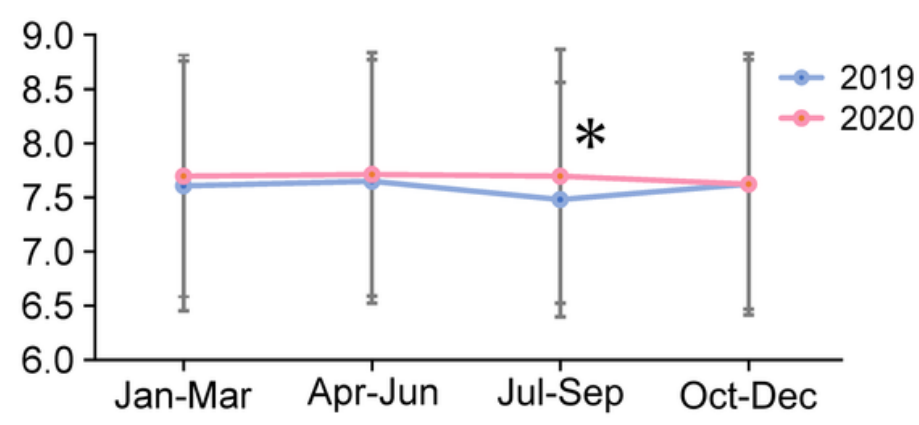

Figure 1

Quarterly obtained HbA1c values in 2019 and 2020
Female

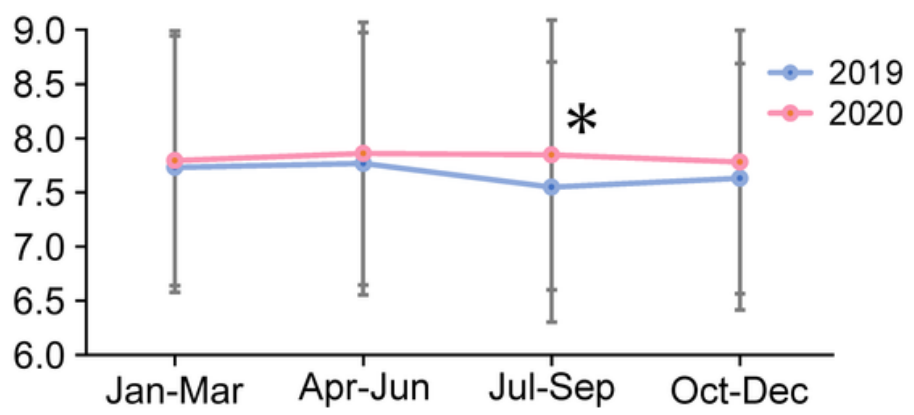




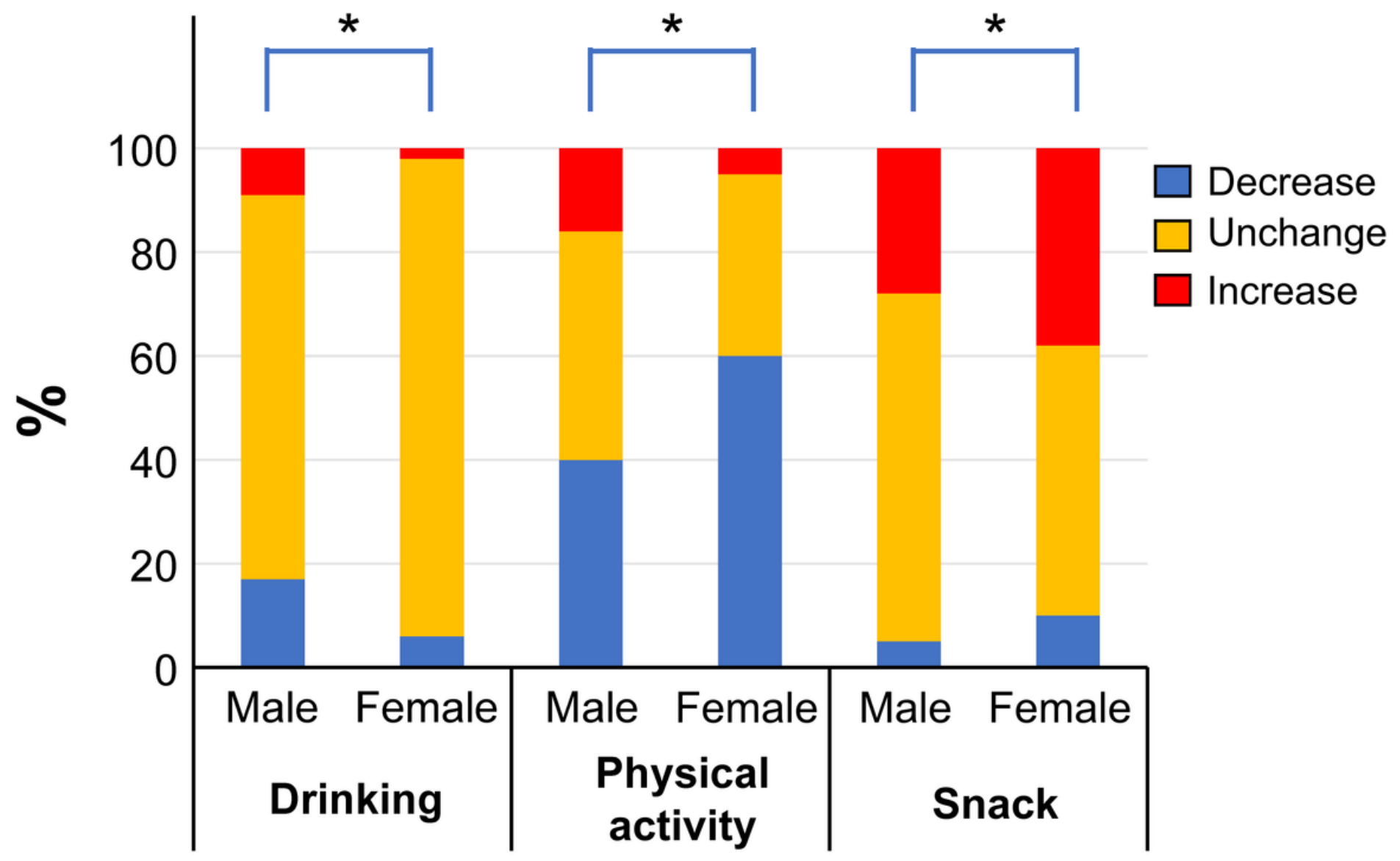

Figure 2

Sex differences in changes in three lifestyle habits 